\title{
Transcriptomic and proteomic profiling revealed reprogramming of carbon metabolism in acetate-grown human pathogen Candida glabrata
}

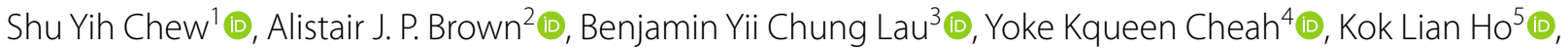
Doblin Sandai ${ }^{6}$, Hassan Yahaya ${ }^{1,7}$ and Leslie Thian Lung Than ${ }^{1 *}$ (i)

\begin{abstract}
Background: Emergence of Candida glabrata, which causes potential life-threatening invasive candidiasis, has been widely associated with high morbidity and mortality. In order to cause disease in vivo, a robust and highly efficient metabolic adaptation is crucial for the survival of this fungal pathogen in human host. In fact, reprogramming of the carbon metabolism is believed to be indispensable for phagocytosed C. glabrata within glucose deprivation condition during infection.
\end{abstract}

Methods: In this study, the metabolic responses of C. glabrata under acetate growth condition was explored using high-throughput transcriptomic and proteomic approaches.

Results: Collectively, a total of 1482 transcripts (26.96\%) and 242 proteins (24.69\%) were significantly up- or downregulated. Both transcriptome and proteome data revealed that the regulation of alternative carbon metabolism in C. glabrata resembled other fungal pathogens such as Candida albicans and Cryptococcus neoformans, with up-regulation of many proteins and transcripts from the glyoxylate cycle and gluconeogenesis, namely isocitrate lyase (ICL1), malate synthase (MLS1), phosphoenolpyruvate carboxykinase (PCK1) and fructose 1,6-biphosphatase (FBP1). In the absence of glucose, C. glabrata shifted its metabolism from glucose catabolism to anabolism of glucose intermediates from the available carbon source. This observation essentially suggests that the glyoxylate cycle and gluconeogenesis are potentially critical for the survival of phagocytosed C. glabrata within the glucose-deficient macrophages.

Conclusion: Here, we presented the first global metabolic responses of C. glabrata to alternative carbon source using transcriptomic and proteomic approaches. These findings implicated that reprogramming of the alternative carbon metabolism during glucose deprivation could enhance the survival and persistence of $C$. glabrata within the host.

Keywords: Candida, Candida glabrata, Acetate, Carbon metabolism, Transcriptomic, Proteomic, RNA-sequencing, Liquid chromatography tandem-mass spectrometry

*Correspondence: leslie@upm.edu.my

1 Department of Medical Microbiology, Faculty of Medicine and Health Sciences, Universiti Putra Malaysia, 43400 Serdang, Selangor, Malaysia

Full list of author information is available at the end of the article

\section{Introduction}

Candida glabrata, an opportunistic human pathogen has become one of the most common etiological agents of invasive candidiasis caused by non-Candida albicans Candida (NCAC) species [1-3]. Multiple studies have underlined the essential role of carbon metabolism in original author(s) and the source, provide a link to the Creative Commons licence, and indicate if changes were made. The images or other third party material in this article are included in the article's Creative Commons licence, unless indicated otherwise in a credit line to the material. If material is not included in the article's Creative Commons licence and your intended use is not permitted by statutory regulation or exceeds the permitted use, you will need to obtain permission directly from the copyright holder. To view a copy of this licence, visit http://creativecommons.org/licenses/by/4.0/. The Creative Commons Public Domain Dedication waiver (http://creativeco mmons.org/publicdomain/zero/1.0/) applies to the data made available in this article, unless otherwise stated in a credit line to the data. 
the survival of pathogenic Candida species [4-7]. These studies suggest that Candida species must be able to utilize a wide range of alternative carbon sources such as lactate and acetate within human host niches. In fact, $C$. glabrata has been reported to be able to assimilate various alternative carbon sources from diverse anatomical sites, including intestinal and vaginal microenvironments $[8,9]$.

It is evident that fungal metabolism, particularly carbon metabolism is of primary importance to human fungal pathogens. In fact, carbon metabolism also affects multiple physiological, immunological and pathogenic attributes of Candida species in glucose-deficient condition [10-12]. In C. albicans, lactate triggers cell wall remodelling that changes the biophysical features and architecture of the fungal cell wall. Furthermore, alteration of these cell wall (and other) properties also increases the stress resistance of C. albicans, including resistance to antifungal drugs, osmotic stress, oxidative stress and cell wall stresses [10]. In contrast, addition of acetic acid to glucose-grown C. glabrata renders the cells to be more sensitive to fluconazole treatment, and these cells are also more susceptible to phagocytosis killing by macrophages [12]. Previously, we have demonstrated that alternative carbon sources also induce physiological changes related to the pathogenicity of C. glabrata [13]. These include changes in planktonic and biofilm growth, cell wall architecture, oxidative stress resistance and antifungal susceptibility.

The "complete" genome sequence of C. glabrata was reported in 2004 [14] and this has proven to be useful in many applications such as transcriptional profiling. Investigations on the global gene expression patterns of fungal pathogens under relevant conditions can potentially assist in the understanding of fungal pathogenesis [15]. Transcriptional analysis of C. glabrata following macrophage and neutrophil engulfment revealed a massive reprogramming response that mirrors the transcriptional landscapes of engulfed C. albicans cells $[4,5,15]$. Metabolic genes involved in gluconeogenic growth such as genes from gluconeogenesis, the glyoxylate cycle and fatty acid $\beta$-oxidation were all significantly up-regulated. In addition, Rai et al. also reported that, during macrophage engulfment, C. glabrata mainly utilizes intermediates from fatty acid degradation through the glyoxylate cycle and gluconeogenesis [7]. Given the fact that alternative carbon sources are relevant in vivo, deciphering the transcriptional and proteomic landscapes of $C$. glabrata in response to these carbon sources could be a key to understand the role of fungal metabolism in shaping the physiological behaviour of C. glabrata.

The arrival of high-throughput next generation sequencing (NGS) technology has paved the way for advanced research that was not possible over a decade ago [16]. In fact, RNA sequencing has been used extensively in understanding of biological and physiological processes of many fungal species [17-20]. In addition, this approach to genome-wide transcript quantification is also recognized to be superior over other transcriptomic methods [21]. As gene expression is also regulated by post-transcriptional modification and translation processes, additional proteomic analysis is also imperative for complete comprehension of the molecular mechanisms [22]. Label-free quantitative proteomic is reproducible and cost effective [23]. This technique is also able to improve sequence coverage and detection of differentially expressed proteins (DEPs) more consistently, compared to the isobaric tagging for relative and absolute quantification (iTRAQ)-based method [24, 25].

Integrating transcriptomic and proteomic analyses is essential for a more complete understanding of the underlying gene regulation, molecular mechanisms and cellular processes of C. glabrata in the presence of physiological relevant alternative carbon source [26]. To achieve this goal, we examined the transcriptional response of C. glabrata grown in media containing acetate as the alternative carbon source by using the highthroughput RNA sequencing. In addition, we performed label-free quantitative proteomic analysis using the liquid chromatography-mass spectrometry. It was anticipated that the comprehensive overview provided by these transcriptome and proteome data might shed light on the metabolic regulation of C. glabrata, particularly under conditions of glucose deprivation.

\section{Materials and methods}

\section{Strain and growth conditions}

Candida glabrata ATCC 2001 (American Type Culture Collection, USA) was used throughout this study. Standard culture media were used, including YPD (Becton, Dickinson and Company, USA): yeast extract $(1 \%, \mathrm{w} / \mathrm{v})$, peptone $(2 \%, \mathrm{w} / \mathrm{v})$, glucose $(2 \%, \mathrm{w} / \mathrm{v})$, agar $(1.5 \%, \mathrm{w} / \mathrm{v})$ and YNB without amino acids (Becton, Dickinson and Company, USA): yeast nitrogen base $(0.67 \%, \mathrm{w} / \mathrm{v})$, ammonium sulfate $(0.5 \%, \mathrm{w} / \mathrm{v})$. Synthetic complete (SC) media were prepared with YNB without amino acids, supplemented with complete supplement mixture $(0.2 \%, \mathrm{w} / \mathrm{v})$ (Formedium, UK), glucose $(2 \%, w / v)$ or acetate $(2 \%, w / v)$ (SigmaAldrich, USA) as the sole carbon source.

\section{RNA extraction}

Briefly, an overnight culture of C. glabrata ATCC 2001 in YPD was washed twice with PBS, pH 7.4, resuspended in fresh YPD $\left(\mathrm{OD}_{600 \mathrm{~nm}}\right.$ of 0.1$)$ and regrown to mid-exponential phase $\left(\mathrm{OD}_{600 \mathrm{~nm}}\right.$ of 0.5$)$. The cell suspension was harvested, washed and resuspended in SC media with $2 \%$ 
$(\mathrm{w} / \mathrm{v})$ glucose or $2 \%(\mathrm{w} / \mathrm{v})$ acetate. Following incubation for $2 \mathrm{~h}$ at $37^{\circ} \mathrm{C}, \mathrm{C}$. glabrata cells were harvested, washed and RNA was extracted using a hot acidic phenol extraction method as previously described [27]. RNA purity and concentration were determined by NanoDrop 1000 spectrophotometer (Thermo Fisher Scientific, USA) and Qubit 2.0 Fluorometer (Thermo Fisher Scientific, USA), respectively. RNA integrity was assessed by Bioanalyzer 2100 using RNA 6000 Nano Kit (Agilent Technologies, USA).

\section{Library construction and RNA-sequencing}

Library construction was carried out using TruSeq RNA Library Prep Kit v2 (Illumina, USA) following manufacturer's recommendations. Briefly, 200 ng of each RNA sample was purified by oligo-dT beads, and poly(A)-containing mRNA was fragmented into small pieces prior to cDNA synthesis. First strand cDNA was generated by First Strand Master Mix and SuperScript II Reverse Transcriptase (Thermo Fisher Scientific, USA), and incubated at $25{ }^{\circ} \mathrm{C}$ for $10 \mathrm{~min} ; 42{ }^{\circ} \mathrm{C}$ for $50 \mathrm{~min} ; 70{ }^{\circ} \mathrm{C}$ for $15 \mathrm{~min}$. Subsequently, Second Strand Master Mix was added to synthesize the second strand $\mathrm{cDNA}$ and incubated at $16{ }^{\circ} \mathrm{C}$ for $1 \mathrm{~h}$. Purified fragmented cDNA was combined with End Repair Mix and incubated at $30^{\circ} \mathrm{C}$ for $30 \mathrm{~min}$. The end-paired DNA was then purified with AMPure $\mathrm{XP}^{\circledR}$ Beads (Beckman Coulter, USA) and incubated with A-Tailing Mix at $37{ }^{\circ} \mathrm{C}$ for $30 \mathrm{~min}$. Adenylated $3^{\prime}$ ends DNA was combined with RNA Index Adapter and Ligation Mix, then the ligation reaction was incubated at $30{ }^{\circ} \mathrm{C}$ for $10 \mathrm{~min}$. The end-paired DNA was purified with AMPure $\mathrm{XP}^{\circledR}$ Beads. Finally, PCR enrichment was performed to enrich the adaptor ligated DNA and the PCR products were purified with AMPure $\mathrm{XP}^{\circledR}$ Beads. The library average molecule length was determined by Bioanalyzer 2100 using DNA 1000 Kit (Agilent Technologies, USA), and the library was quantified via real-time PCR. The final library was amplified within the flow cell on the cBot instrument (Illumina, USA) for cluster generation using HiSeq ${ }^{\circledR} 4000$ PE Cluster Kit (Illumina, USA). Finally, the clustered flow cell was loaded onto the HiSeq 4000 Sequencing System (Illumina, USA) for paired-end sequencing using HiSeq ${ }^{\circledR} 4000$ SBS Kit (Illumina, USA). In total, the experiments were performed in triplicates using C. glabrata grown in two different growth conditions (glucose and acetate).

\section{Reads mapping and differential expression analysis}

Quality of the raw sequencing reads was accessed by FastQC v0.10.1. Prior to reads mapping, low quality reads and bases (quality value $<30$ and length size $<50 \mathrm{bp}$ ), ambiguous bases and artefacts were removed by FastX toolkit v0.0.13.2. Orphan reads obtained after the trimming and filtering were also removed. Clean reads were mapped to the reference genome of C. glabrata CBS 138 (GCF_000002545.3). The reads mapping was carried out using TopHat v2.0.12 [28], which used Bowtie2 tool as algorithmic core, by allowing up to two reads mismatches. The expression of genes annotated in reference genome were quantified using Cufflinks v2.2.1. Differentially expressed genes between glucose-grown C. glabrata (control) and acetate-grown C. glabrata were analyzed by Cuffdiff from the Cufflinks package [29]. The coverage of reads mapped was translated to expression value which represented in fragments per kilobase of transcript per million mapped fragments (FPKM) [29]. Statistically significant DEGs were called with arbitrary cut-off values of $\log 2$ fold change $\geq 1.5$ and $q$ value of $\leq 0.05$, with false discovery rate (FDR) less than 5\%. RNA-sequencing data are available at European Nucleotide Archive (ENA) [30] under the study accession number PRJEB33880.

\section{Functional annotation and enrichment analysis}

Functional annotation and enrichment analyses of DEGs were performed using Database for Annotation, Visualization and Integrated Discovery (DAVID) Bioinformatics Resource 6.8 tool (https://david.ncifcrf.gov/) [31] with DAVID-defined default annotation categories. Enrichment analysis of Gene ontology (GO) was performed to identify and categorize enriched biological process, molecular function and cellular component. In addition, DEGs were also searched against the Kyoto Encyclopedia of Genes and Genomes (KEGG) database to detect significantly enriched pathways.

\section{Validation of RNA-sequencing data}

Validation of RNA sequencing data was performed by quantification of gene expression via quantitative realtime PCR (qPCR). Total RNA was reversibly transcribed to cDNA using Maxima $\mathrm{H}$ Minus First Strand cDNA Synthesis Kit (Thermo Scientific, USA), followed by PCR amplification with SensiFAST SYBR ${ }^{\circledR}$ No-ROX Kit (Bioline, UK). Fold changes in relative gene expression were determined by the changes in cycle threshold (CT) values and data obtained were normalized against the $\beta$-actin transcript, ACT1. Relative fold change was calculated using delta-delta $\mathrm{CT}$ method $\left(2^{-\Delta \Delta \mathrm{CT}}\right)$. Primers used were described in Additional file 1: Table S1. Primers used in this study were designed by using the free online Primer-BLAST software from National Centre for Biotechnology Information (NCBI). All primers were synthesized commercially by Integrated DNA Technologies, USA.

\section{Protein extraction}

Protein extraction was performed as previously described with slight modification [32]. An overnight cultures of $C$. 
glabrata ATCC 2001 in YPD was harvested and washed twice with PBS, pH 7.4 before resuspended into fresh $\mathrm{SC}$ media $\left(\mathrm{OD}_{600 \mathrm{~nm}}\right.$ of 0.1$)$ supplemented with $2 \%(\mathrm{w} / \mathrm{v})$ glucose or $2 \%(\mathrm{w} / \mathrm{v})$ acetate. Following incubation for $24 \mathrm{~h}$ at $37^{\circ} \mathrm{C}$, the $C$. glabrata cells were centrifuged at $4000 \times g$ for $5 \mathrm{~min}$ and resuspended in $1 \mathrm{ml}$ of lysis buffer consist of $7 \mathrm{M}$ urea (Bio-Rad Laboratories, USA), 4\% (w/v) 3-[(3-cholamidopropyl)dimethylammonio]-1-propanesulfonate (CHAPS) (Bio-Rad Laboratories, USA), $0.4 \%(\mathrm{w} / \mathrm{v})$ dithiothreitol (DTT) (Bio-Rad Laboratories, USA) and 1 tablet of protease inhibitor cocktail (Roche Diagnostics GmbH, Germany). The cell suspension was mixed with equal volume of $0.5 \mathrm{~mm}$ glass beads (BDH Chemicals, VMR, USA) and disrupted mechanically with FastPrep-24 ${ }^{\mathrm{TM}}$ instrument (MP Biomedicals, USA) at the speed of $6 \mathrm{M} / \mathrm{s}$ for 2 cycles of $30 \mathrm{~s}$ with short icecold incubations in between. Following centrifugation at $3000 \times g$ for $5 \mathrm{~min}$, proteins in the supernatant were precipitated by adding five volumes of $0.1 \mathrm{M}$ ice-cold ammonium acetate in methanol (Merck, Germany) and incubated overnight at $-20{ }^{\circ} \mathrm{C}$. The protein pellet was collected by centrifugation at $15,000 \times g$ for $15 \mathrm{~min}$ at $4{ }^{\circ} \mathrm{C}$ and washed twice with ice-cold $80 \%$ (v/v) acetone (Merck, Germany). Finally, the protein pellet was airdried for $5 \mathrm{~min}$ and resuspended in $50 \mathrm{mM}$ ammonium bicarbonate (Sigma-Aldrich, USA) containing $1 \mathrm{M}$ urea. The protein concentrations in each sample was quantified with Pierce $660 \mathrm{~nm}$ protein assay reagent (Thermo Fisher Scientific, USA).

\section{In-solution protein digestion}

In-solution protein digestion was performed according to a modified procedure previously described [33]. Prior to protein digestion, protein samples were reduced with $100 \mathrm{mM}$ tris(2-carboxyethyl) phosphine (TCEP) (SigmaAldrich, USA) in $50 \mathrm{mM}$ ammonium bicarbonate for $1 \mathrm{~h}$ at $60{ }^{\circ} \mathrm{C}$, followed by alkylation using $200 \mathrm{mM}$ iodoacetamide (Bio-Rad Laboratories, USA) in $50 \mathrm{mM}$ ammonium bicarbonate for $45 \mathrm{~min}$ at room temperature. Subsequently, $1 \%(\mathrm{w} / \mathrm{v})$ sodium deoxycholate (Sigma-Aldrich, USA) in $5 \mathrm{mM}$ ammonium bicarbonate was added and the protein samples were incubated for $10 \mathrm{~min}$ at $37^{\circ} \mathrm{C}$. Protein samples were mixed with modified sequencing grade trypsin (Promega, USA) in a ratio of $1 \mu \mathrm{g}$ trypsin to $50 \mu \mathrm{g}$ protein, $10 \%(\mathrm{v} / \mathrm{v})$ acetonitrile (Merck, Germany) in $5 \mathrm{mM}$ ammonium bicarbonate, was added before the mixture was incubated for $16 \mathrm{~h}$ at $37^{\circ} \mathrm{C}$. Sodium deoxycholate was removed by incubating the peptide samples with $0.5 \%(\mathrm{v} / \mathrm{v})$ formic acid (Fisher Scientific, USA) for $45 \mathrm{~min}$ at $37{ }^{\circ} \mathrm{C}$. Peptide samples were centrifuged at $14,000 \times g$ for $15 \mathrm{~min}$ and dried in a vacuum concentrator (Concentrator Plus, Eppendorf, Germany). Dried peptide pellet was resuspended in $100 \mu \mathrm{l}$ of $0.1 \%(\mathrm{v} / \mathrm{v})$ formic acid. Acetonitrile-washed and methanol-conditioned Empore solid phase extraction (SPE) C18 disks (3 M Science, USA) was added to the resuspended pellet and incubated for $3 \mathrm{~h}$ at room temperature with mild agitation. Following $3 \mathrm{~h}$ of incubation, C18 disks were removed from the peptide samples and the bound peptides were eluted by using $100 \mu \mathrm{l}$ of elution buffer $(50 \%$ acetonitrile in $0.1 \%$ (v/v) formic acid) for $30 \mathrm{~min}$. The eluent was transferred to a clean microcentrifuge tube and dried with the vacuum concentrator.

\section{Liquid chromatography tandem-mass spectrometry}

For mass spectrometric analysis, peptide digests were reconstituted in $30 \mu \mathrm{l}$ of $0.1 \%(\mathrm{v} / \mathrm{v})$ formic acid and $5 \%(\mathrm{v} / \mathrm{v})$ acetonitrile prior to sample loading. A nanoflow ultra-high-performance liquid chromatography (UHPLC) instrument (EASY-nLC 1200 System, Thermo Fisher Scientific, USA) coupled online to a Q Exactive Plus Quadrupole-Orbitrap mass spectrometer (Thermo Fisher Scientific, USA) with a Nanospray Flex ion source was used for analysis. In total, the experiments were performed in duplicates using C. glabrata grown in two different growth conditions (glucose and acetate). Chromatography column used for separation was Acclaim PepMap 100 C18 (Thermo Fisher Scientific, USA). Approximately $2 \mu \mathrm{g}$ of the peptide mixture was loaded onto the reversed phase column $(15 \mathrm{~cm}$ long, $75 \mu \mathrm{m}$ inner diameter) and separated with a linear gradient of $5-50 \%$ Buffer B (80\% (v/v) acetonitrile and $0.1 \%(\mathrm{v} / \mathrm{v})$ for$\mathrm{mic}$ acid) at a flow rate of $300 \mathrm{nl} / \mathrm{min}$ for $90 \mathrm{~min}$.

The spray voltage was set to $1.96 \mathrm{kV}$, funnel radial force level at 50, and heated capillary at $275^{\circ} \mathrm{C}$. The Q Exactive Plus was configured for data-dependent acquisition using the full MS/DD-MS/MS setup. Top15 method dynamically chose 15 of the most abundant precursor ions from the survey scan for high-energy collisional dissociation (HCD) fragmentation. Full MS resolution was set to 70,000 at $\mathrm{m} / z 200$ and full MS automatic gain control (AGC) target was 3e6 with a maximum injection time of $100 \mathrm{~ms}$. Mass range was set to $310-1800 \mathrm{~m} / z$. Resolution for HCD spectra was set to 17,500 at $m / z 200$. AGC target value for fragment spectra was set at $1 \mathrm{e} 5$, intensity threshold was kept at $8.3 \mathrm{e} 4$ and maximum injection time was set to $60 \mathrm{~ms}$. Isolation width was set at $0.7 \mathrm{~m} / \mathrm{z}$. Normalised collision energy was set at $27 \%$. Peptide match was set to preferred, and isotope exclusion was on. All data were acquired in profile mode using positive polarity.

\section{Data processing and protein identification}

Mass spectrometry data obtained were analyzed with the Proteome Discoverer version 2.2 (Thermo Fisher Scientific, USA) using the integrated SEQUEST search engine 
against the Candida UniProt protein database (September 2018 release, 231,653 sequence entries). Trypsin was specified as the enzyme, cleaving after lysine and arginine residues and allowing up to two missed cleavages. Carbamidomethylation of cysteine was set as fixed modification and $\mathrm{N}$-terminal protein acetylation, methionine oxidation, asparagine and glutamine deamidation as variable or dynamic modifications. Proteome Discoverer was used to score peptides for identification based on a search with an initial allowed mass deviation of the precursor ion of up to $10 \mathrm{ppm}$. The allowed fragment mass deviation was $0.02 \mathrm{Da}$. The FDR was set to 0.01 for proteins and peptides, which had to have a minimum length of 6 amino acids. To further reduce the false peptide identifications, percolator algorithm was applied to discriminate between the correct and incorrect peptide spectrum matches based on probability and q value. All subsequent statistical analysis was performed using the protein and peptide level data using Proteome Discoverer. Peak intensities were normalized and subjected to the principal component analysis. Volcano plot was used to visualize the expression changes. The plot was derived from the protein/peptide abundance ratios and $p$ values. The mass spectrometry proteomics data have been deposited to the ProteomeXchange Consortium via the PRIDE [34] partner repository with the dataset identifier PXD014916 and 10.6019/PXD014916.

\section{Results}

Transcriptomic landscape of acetate-grown C. glabrata

Comparative RNA sequencing was employed to identify the differential gene expression between acetate-grown $C$. glabrata and glucose-grown C. glabrata (control). In general, approximately 65 million raw reads were obtained from transcriptome for all six samples (each growth condition in triplicate). Following the process of quality filtering, over $98 \%$ of the sequencing data were retained and less than $0.5 \%$ reads were removed as orphan reads
(Table 1). High quality cleaned reads were then mapped to the reference genome of C. glabrata CBS 138, and high percentage of the reads (93\%) were uniquely mapped to the selected genome. In addition, high correlations were observed among the three biological replicates for the glucose-grown C. glabrata $\left(0.94<\mathrm{r}^{2}<0.95\right)$ and acetategrown C. glabrata $\left(0.95<\mathrm{r}^{2}<0.96\right)$ (Additional file 1 : Table S2).

The transcriptome of the acetate-grown C. glabrata was compared to glucose-grown C. glabrata to calculate the differential gene expression. A cut-off of 1.5 -fold change and $q$ value $<0.05$ were applied to determine the statistically significant up- and down-regulated genes. Overall, approximately $93 \%$ out of 5498 genes were found to be reliably expressed in both conditions, among which $1482(26.96 \%)$ were significantly expressed in acetategrown C. glabrata compared to glucose. Among these 1482 DEGs, 744 (13.53\%) were significantly up-regulated and $732(13.31 \%)$ were significantly down-regulated.

\section{Verification of RNA-sequencing data}

Quantitative real-time PCR was performed to test the validity of the results obtained from the RNA sequencing analysis. Fifteen DEGs were chosen for qPCR analysis, which included eight up-regulated genes: isocitrate lyase (ICL1), malate synthase (MLS1), phosphoenolpyruvate carboxykinase (PCK1), fructose 1,6-bisphosphatase (FBP1), putative plasma membrane high affinity glucose sensor (SNF3), citrate synthase (CIT1), C6 zinc cluster transcriptional activator (SIP4), putative zinc cluster protein (GSM1) and seven down-regulated genes: pyruvate carboxylase (PYC2), pyruvate kinase (PYK1), phosphofructokinase (PFK1), 6-phosphofructokinase (PFK2), 6-phosphofructo-2-kinase (PFK27), enolase (ENO1), glyceraldehyde-3-phosphate dehydrogenase (TDH3) in the acetate-grown cells, relative to the control glucose-grown cells. Expression of all DEGs obtained

Table 1 Summary of the transcriptome data and genome mapping from glucose- and acetate-grown C. glabrata samples

\begin{tabular}{|c|c|c|c|c|c|c|}
\hline \multirow[t]{2}{*}{ Sample group } & \multicolumn{3}{|c|}{ Glucose-grown C. glabrata } & \multicolumn{3}{|c|}{ Acetate-grown C. glabrata } \\
\hline & Glu 1 & Glu 2 & Glu 3 & Ace 1 & Ace 2 & Ace 3 \\
\hline Total raw reads & $11,167,982$ & $11,067,988$ & $11,108,556$ & $11,026,716$ & $10,713,412$ & $10,502,714$ \\
\hline Total clean reads & $11,069,487$ & $10,968,492$ & $11,012,892$ & $10,927,921$ & $10,615,974$ & $10,400,162$ \\
\hline Paired reads & $11,035,394$ & $10,934,808$ & $10,978,978$ & $10,894,900$ & $10,583,306$ & $10,368,454$ \\
\hline Orphan reads & 34,093 & 33,684 & 33,914 & 33,021 & 32,668 & 31,708 \\
\hline GC content (\%) & 44 & 45 & 45 & 45 & 43 & 44 \\
\hline Reads uniquely mapped & $10,260,577$ (93.0\%) & $10,186,208(93.1 \%)$ & $10,292,990$ (93.7\%) & $10,177,516(93.4 \%)$ & $9,997,771(94.5 \%)$ & $9,733,441(93.9 \%)$ \\
\hline Reads multiply mapped & $380,256(3.4 \%)$ & $325,515(3.0 \%)$ & $315,263(2.9 \%)$ & $163,869(1.5 \%)$ & $137,062(1.3 \%)$ & $147,681(1.4 \%)$ \\
\hline Reads unmapped & $394,561(3.6 \%)$ & $423,085(3.9 \%)$ & $370,725(3.4 \%)$ & $553,515(5.1 \%)$ & $448,473(4.2 \%)$ & $487,332(4.7 \%)$ \\
\hline
\end{tabular}



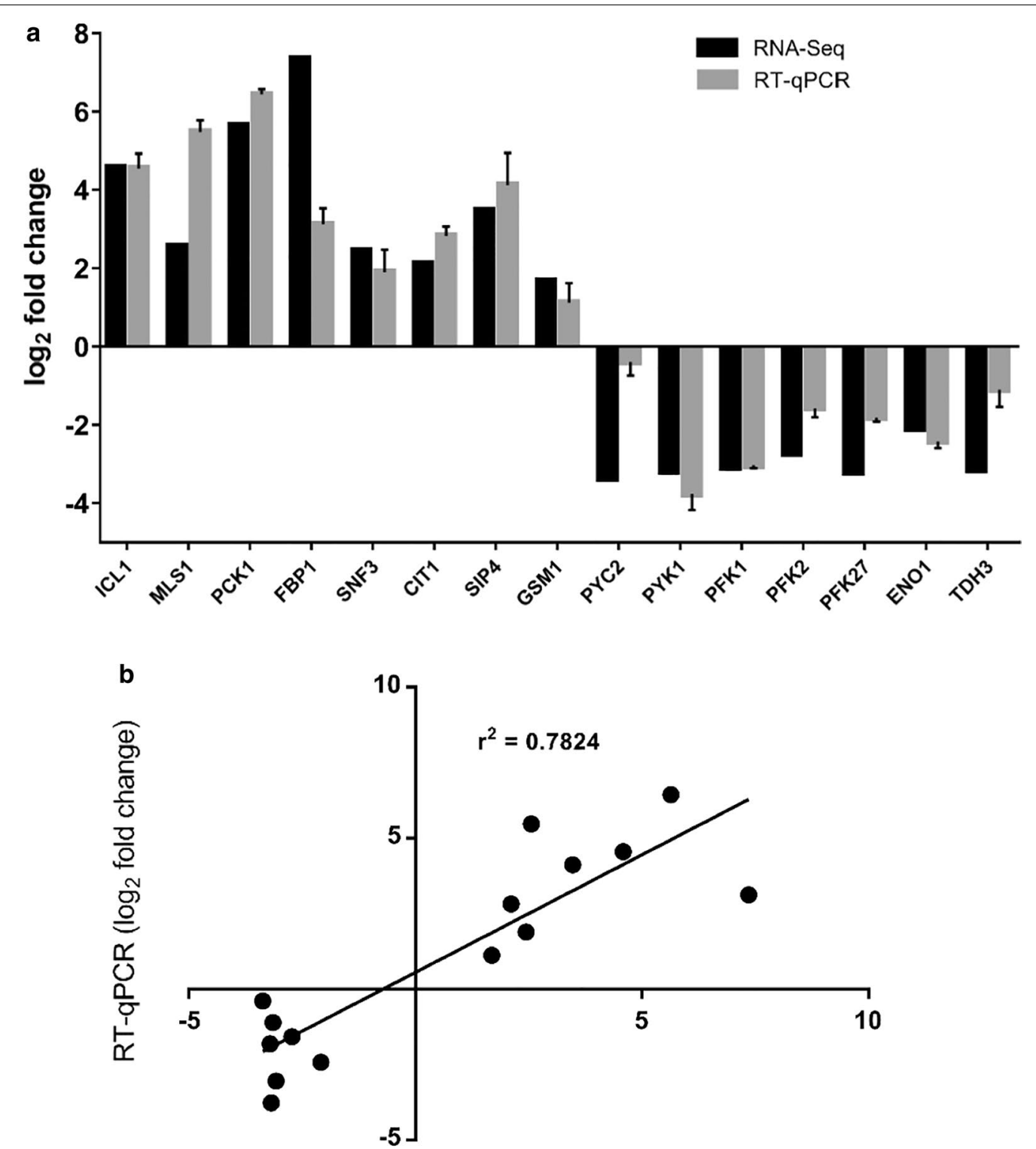

RNA-Seq ( $\log _{2}$ fold change)

Fig. 1 Verification of the RNA sequencing data. a Comparison of gene expression levels of 15 DEGs between RNA sequencing and RT-qPCR analyses. $\mathbf{b}$ Correlation between gene expression levels of 15 DEGs obtained from RNA sequencing and RT-qPCR analyses. Statistically significant Pearson correlation $\left(r^{2}=0.7824\right)$ was observed between the two quantitative methods

from $\mathrm{qPCR}$ analysis were in agreement with results from the RNA sequencing analysis (Fig. 1a). Although the correlation was not so strong on PYC2, both RNA sequencing and qPCR data still indicated this gene as down-regulated. Results from RNA sequencing analysis and qPCR also demonstrated significant Pearson correlations $\left(r^{2}=0.7824\right)$, further confirming the reliability of the transcriptome data (Fig. 1b).

\section{Functional enrichment of the DEGs}

Functional enrichment analysis was performed on upand down-regulated DEGs to detect significant enriched GO and KEGG pathways according to DAVID Bioinformatics Resource 6.8. A total of 744 up-regulated and 732 down-regulated DEGs (in the acetate-, compared to the control glucose-grown cells) were assigned into three GO categories: biological process, cellular component and 
molecular function. In total, $66 \mathrm{GO}$ terms were found to be enriched in up-regulated DEGs [expression analysis systematic explorer (EASE) score $<0.1$ ], of which 31 were involved with biological process, 21 with molecular function and 14 with cellular compartment (Additional file 1: Table S3). Besides, among the 46 GO terms enriched in down-regulated DEGs (EASE score $<0.1$ ), 23 were involved with biological process, 13 with molecular function and 10 with cellular compartment (Additional file 1: Table S4).

Additionally, 10 and 30 KEGG pathways were found associated with the up-regulated and down-regulated DEGs, respectively. Notably, the genes up-regulated in acetate were involved in pathways associated with oxidative phosphorylation, mitogen-activated protein kinase (MAPK) signalling pathway, tricarboxylic acid (TCA) cycle, nucleotide excision repair, peroxisome, homologous recombination, endocytosis, protein processing in endoplasmic reticulum, carbon metabolism, glyoxylate and dicarboxylate metabolism (Table 2). Meanwhile, the down-regulated genes in acetate were primarily involved in pathways associated with biosynthesis of amino acids, biosynthesis of secondary metabolites, biosynthesis of antibiotics, as well as carbon metabolism and glycolysis (Table 2).

\section{Differential expressed genes associated with carbon metabolism in acetate-grown C. glabrata cells}

In the present study, the expression of high numbers of carbon metabolism genes were altered in acetate-grown C. glabrata. In the presence of acetate, glycolytic genes such as PFK1, PFK2, TDH3, ENO1, 3-phosphoglycerate kinase (PGK1), and pyruvate kinase (CDC19) were all down-regulated, ranging from 2.03-fold to 3.15-fold (Table 3). Although most of the reactions and enzymes are shared between glycolysis and the gluconeogenesis, two enzyme genes exclusive to gluconeogenesis (FBP1, PCK1) were significantly up-regulated 7.35-fold and 5.64-fold, respectively (Table 3). In addition, metabolic enzyme genes from the glyoxylate cycle were upregulated in acetate-grown $C$. glabrata cells (Table 3 ). These include ICL1 (4.58-fold), MLS1 (2.56-fold), CIT1 (2.11-fold) and putative aconitate hydratase (ACO1) (1.63-fold). Also, genes encoding TCA cycle enzymes such as isocitrate dehydrogenase (IDP2), $\alpha$-ketoglutarate dehydrogenase complex (KGD1, KGD2), succinate dehydrogenases (SDH1, SDH2 and SHD4) were significantly up-regulated (Table 3).

\section{Proteomic landscape of acetate-grown C. glabrata}

To investigate the impact of alternative carbon source on the proteome of C. glabrata, cells were exposed to
$2 \%(\mathrm{w} / \mathrm{v})$ glucose or $2 \%(\mathrm{w} / \mathrm{v})$ acetate for $24 \mathrm{~h}$ and analyzed with label-free quantitative proteomics. A total of three technical replicates for each of the two biological replicates of the growth conditions were analyzed. As shown in Fig. 2, majority of the separated peptides had masses between 300 and $1500 \mathrm{Da}$, with most of the peptides eluting from 10 to $80 \mathrm{~min}$. On the other hand, highly abundance proteins (e.g. glyceraldehyde-3-phosphate dehydrogenase, enolase and phosphoglycerate kinase) identified were up to 5-log higher abundance in comparison to the lowest abundance proteins (e.g. proteasome subunit beta type 2 and putative ubiquitin-conjugating enzyme), indicating a good and acceptable protein detection range in both growth conditions (Fig. 3).

In the present study, label-free quantitative proteomic analysis uncovered 980 proteins, with only a limited amount of proteins identified "exclusively" under glucose- or acetate-grown conditions (note that "exclusive" expression meant that the level of a given protein was below the detection limit for this analysis under one condition, rather than not being expressed under that condition). A total of 106 proteins were expressed exclusively in acetate-grown C. glabrata cells, while only 19 proteins were exclusive to the glucose-grown cells (Fig. 4). Hierarchical clustering analysis demonstrated a clear difference in the proteomes of the glucose-grown and acetate-grown C. glabrata cells, with only minimal variance between the biological replicates (Fig. 5). Although the majority of identified proteins were not significantly regulated, a small portion of identified proteins (192 significantly up-regulated, 50 significantly down-regulated in acetate) was differentially expressed between the two growth conditions ( $\log 2$ fold change $>1.5$ and $\mathrm{q}$ value $<0.05)$.

\section{Functional enrichment of the DEPs}

Functional enrichment analysis was also performed on the DEPs to detect the significantly enriched GO and KEGG pathways using DAVID Bioinformatics Resource 6.8 tool. A total of 22 and two GO terms were strongly enriched in the up- and down-regulated proteins in acetate, respectively, with EASE Score less than 0.1 (Additional file 1: Table S5). Pathway analysis revealed that 14 and four KEGG pathways were also significantly enriched (Table 4). Proteins associated with metabolic pathways were the most represented in acetate-grown C. glabrata. This included pathways involved in oxidative phosphorylation, carbon metabolism, citrate cycle, pyruvate metabolism, 2-oxocarboxylic acid metabolism, gluconeogenesis, glyoxylate and dicarboxylate metabolism. 
Table 2 List of enriched Kyoto encyclopedia of genes and genomes (KEGG) pathways in the differentially expressed genes (DEG)s of acetate-grown C. glabrata cells

\begin{tabular}{|c|c|c|c|c|}
\hline KEGG entry & Pathway & Count & Frequency (\%) & EASE score \\
\hline \multicolumn{5}{|c|}{ Up-regulated DEGs } \\
\hline cgr00190 & Oxidative phosphorylation & 25 & 3.36 & $5.76 \mathrm{E}-09$ \\
\hline cgr04011 & MAPK signalling pathway—yeast & 13 & 1.75 & $2.54 \mathrm{E}-04$ \\
\hline cgr00020 & Citrate cycle (TCA cycle) & 9 & 1.21 & 0.006 \\
\hline cgr03420 & Nucleotide excision repair & 10 & 1.34 & 0.0073 \\
\hline cgr04146 & Peroxisome & 10 & 1.34 & 0.0073 \\
\hline cgr03440 & Homologous recombination & 7 & 0.94 & 0.011 \\
\hline cgr04144 & Endocytosis & 14 & 1.88 & 0.0133 \\
\hline cgr04141 & Protein processing in endoplasmic reticulum & 14 & 1.88 & 0.047 \\
\hline cgr00630 & Glyoxylate and dicarboxylate metabolism & 6 & 0.81 & 0.0519 \\
\hline cgr01200 & Carbon metabolism & 16 & 2.15 & 0.0596 \\
\hline \multicolumn{5}{|c|}{ Down-regulated DEGs } \\
\hline cgr01230 & Biosynthesis of amino acids & 69 & 9.43 & 8.67E-29 \\
\hline cgr01100 & Metabolic pathways & 180 & 24.59 & $2.96 \mathrm{E}-28$ \\
\hline cgr01110 & Biosynthesis of secondary metabolites & 100 & 13.66 & $5.22 \mathrm{E}-21$ \\
\hline cgr01130 & Biosynthesis of antibiotics & 70 & 9.56 & $6.25 E-13$ \\
\hline cgr01210 & 2-Oxocarboxylic acid metabolism & 23 & 3.14 & $2.32 \mathrm{E}-10$ \\
\hline cgr00260 & Glycine, serine and threonine metabolism & 18 & 2.46 & $1.28 \mathrm{E}-07$ \\
\hline cgr00300 & Lysine biosynthesis & 11 & 1.5 & $4.06 \mathrm{E}-07$ \\
\hline cgr00290 & Valine, leucine and isoleucine biosynthesis & 10 & 1.37 & $7.62 \mathrm{E}-06$ \\
\hline cgr00400 & Phenylalanine, tyrosine and tryptophan biosynthesis & 12 & 1.64 & $1.41 \mathrm{E}-05$ \\
\hline cgr00270 & Cysteine and methionine metabolism & 16 & 2.19 & $4.55 E-05$ \\
\hline cgr00920 & Sulfur metabolism & 8 & 1.09 & $4.20 \mathrm{E}-04$ \\
\hline cgr00450 & Selenocompound metabolism & 7 & 0.96 & 0.0017 \\
\hline cgr00100 & Steroid biosynthesis & 9 & 1.23 & 0.0023 \\
\hline cgr00670 & One carbon pool by folate & 8 & 1.09 & 0.0071 \\
\hline cgr00510 & $\mathrm{N}$-Glycan biosynthesis & 11 & 1.5 & 0.0099 \\
\hline cgr00514 & Other types of O-glycan biosynthesis & 6 & 0.82 & 0.012 \\
\hline cgr00564 & Glycerophospholipid metabolism & 12 & 1.64 & 0.0184 \\
\hline cgr00680 & Methane metabolism & 9 & 1.23 & 0.0195 \\
\hline cgr00010 & Glycolysis/gluconeogenesis & 14 & 1.91 & 0.0251 \\
\hline cgr00340 & Histidine metabolism & 6 & 0.82 & 0.0287 \\
\hline cgr00770 & Pantothenate and CoA biosynthesis & 7 & 0.96 & 0.0289 \\
\hline cgr00220 & Arginine biosynthesis & 7 & 0.96 & 0.0289 \\
\hline cgr00460 & Cyanoamino acid metabolism & 4 & 0.55 & 0.0303 \\
\hline cgr00230 & Purine metabolism & 21 & 2.87 & 0.0405 \\
\hline cgr01200 & Carbon metabolism & 23 & 3.14 & 0.0468 \\
\hline cgr00860 & Porphyrin and chlorophyll metabolism & 7 & 0.96 & 0.0508 \\
\hline cgr00261 & Monobactam biosynthesis & 3 & 0.41 & 0.0665 \\
\hline cgr00660 & C5-Branched dibasic acid metabolism & 3 & 0.41 & 0.0665 \\
\hline cgr00051 & Fructose and mannose metabolism & 7 & 0.96 & 0.0808 \\
\hline cgr00240 & Pyrimidine metabolism & 16 & 2.19 & 0.0929 \\
\hline
\end{tabular}

Interestingly, KEGG pathways associated with biosynthesis of secondary metabolites, antibiotics and amino acids were detected as up-regulated at the protein level only (Table 4).
Differential expressed proteins associated with carbon metabolism in acetate-grown C. glabrata cells

A large portion of the proteomic response in acetategrown C. glabrata involved the increase abundance of proteins associated with carbon metabolism, particularly 
Table 3 Statistically significant differential gene expressions associated with carbon metabolism in acetate-grown $C$. glabrata (q value $<0.05$ )

\begin{tabular}{|c|c|c|c|}
\hline Systematic name & Genes & Description & $\begin{array}{l}\begin{array}{l}\text { Fold } \\
\text { change } \\
(\log 2)\end{array}\end{array}$ \\
\hline \multicolumn{4}{|c|}{ Up-regulated genes associated with carbon metabolism in acetate-grown C. glabrata } \\
\hline CAGLOH04939G & FBP1 & Fructose 1,6-bisphosphatase & 7.35 \\
\hline CAGLOH06633G & PCK1 & Phosphoenolpyruvate carboxykinase & 5.64 \\
\hline CAGLOJ03058G & ICL1 & Isocitrate lyase & 4.58 \\
\hline CAGLOC03223G & $\mathrm{SDH} 2$ & Succinate dehydrogenase & 4.16 \\
\hline CAGLOM06963G & SOL2 & Suppressor of Los1-1 & 2.68 \\
\hline CAGLOL03982G & MLS1 & Malate synthase & 2.56 \\
\hline CAGLOG08712G & KGD1 & a-Ketoglutarate dehydrogenase complex & 2.51 \\
\hline CAGLOH03993G & CIT1 & Citrate synthase & 2.11 \\
\hline CAGLOF05863G & $\mathrm{SDH} 4$ & Succinate dehydrogenase & 1.98 \\
\hline CAGL0J00847G & SDH1 & Succinate dehydrogenase & 1.92 \\
\hline CAGLOL03740G & RKI1 & Ribose-5-phosphate isomerase & 1.92 \\
\hline CAGLOB04917G & IDP2 & Isocitrate dehydrogenase & 1.89 \\
\hline CAGLOE01287G & KGD2 & a-Ketoglutarate dehydrogenase complex & 1.73 \\
\hline CAGL0D06424G & ACO1 & Putative aconitate hydratase & 1.63 \\
\hline CAGLOK10868G & CTA1 & Putative catalase A & 1.60 \\
\hline \multicolumn{4}{|c|}{ Down-regulated genes associated with carbon metabolism in acetate-grown C. glabrata } \\
\hline CAGLOK00825G & SER2 & Phosphoserine phosphatase & -3.52 \\
\hline CAGLOI09284G & SHM1 & Glycine hydroxymethyltransferase & -3.42 \\
\hline CAGLOK06787G & PYC2 & Pyruvate carboxylase & -3.37 \\
\hline CAGLOL11088G & YOR283W & Phosphatase & -3.25 \\
\hline CAGLOM12034G & CDC19 & Pyruvate kinase & -3.18 \\
\hline CAGL0J00451G & $\mathrm{TDH} 3$ & Glyceraldehyde-3-phosphate dehydrogenase & -3.15 \\
\hline CAGLOK08580G & AAT1 & Aspartate aminotransferase & -3.11 \\
\hline CAGLOF08041G & PFK1 & Phosphofructokinase & -3.08 \\
\hline CAGLOH05137G & ALD5 & Aldehyde dehydrogenase & -2.90 \\
\hline CAGLOL10758G & PFK2 & 6-Phosphofructokinase & -2.73 \\
\hline CAGLOI05126G & ILVI & L-Threonine ammonia-lyase & -2.56 \\
\hline CAGLOI05500G & PRS2 & Ribose phosphate diphosphokinase & -2.28 \\
\hline CAGLOC05181G & PRS5 & Ribose phosphate diphosphokinase & -2.29 \\
\hline CAGLOE06358G & GPM1 & Phosphoglycerate mutase & -2.24 \\
\hline CAGLOI02486G & ENO1 & Enolase & -2.09 \\
\hline CAGLOH08327G & TPI1 & Triose-phosphate isomerase & -2.07 \\
\hline CAGL0D06402G & MET15 & O-Acetyl homoserine sulfhydrylase & -2.05 \\
\hline CAGLOL07722G & PGK1 & 3-Phosphoglycerate kinase & -2.03 \\
\hline CAGLOL03311G & SHB17 & Sedoheptulose-bisphosphatase & -2.01 \\
\hline CAGLOD04356G & GCV1 & Glycine dehydrogenase & -1.96 \\
\hline CAGLOF01749G & SHM2 & Serine hydroxymethyltransferase & -1.94 \\
\hline CAGLOH07579G & HXK2 & Hexokinase & -1.84 \\
\hline CAGL0J01441G & $A D H 2$ & Alcohol dehydrogenase & -1.55 \\
\hline CAGL0J09504G & MET12 & Methylenetetrahydrofolate reductase & -1.51 \\
\hline
\end{tabular}

Data of differential gene expressions ( $q$ value $<0.05$ ) was obtained from RNA-sequencing analysis. Fold change $(\log 2)$ was calculated based on the gene expression of acetate-grown C. glabrata to glucose-grown C. glabrata (control)

in gluconeogenesis and the glyoxylate cycle (Table 5). Notably, glyoxylate cycle enzymes, which include isocitrate lyase (6.64-fold), malate synthase (2.59-fold), malate dehydrogenase (1.53-fold), citrate synthase (3.30-fold) and aconitase hydratase (6.64-fold), succinate dehydrogenase (2.62-fold) and fumarate hydratase (1.60-fold), were 

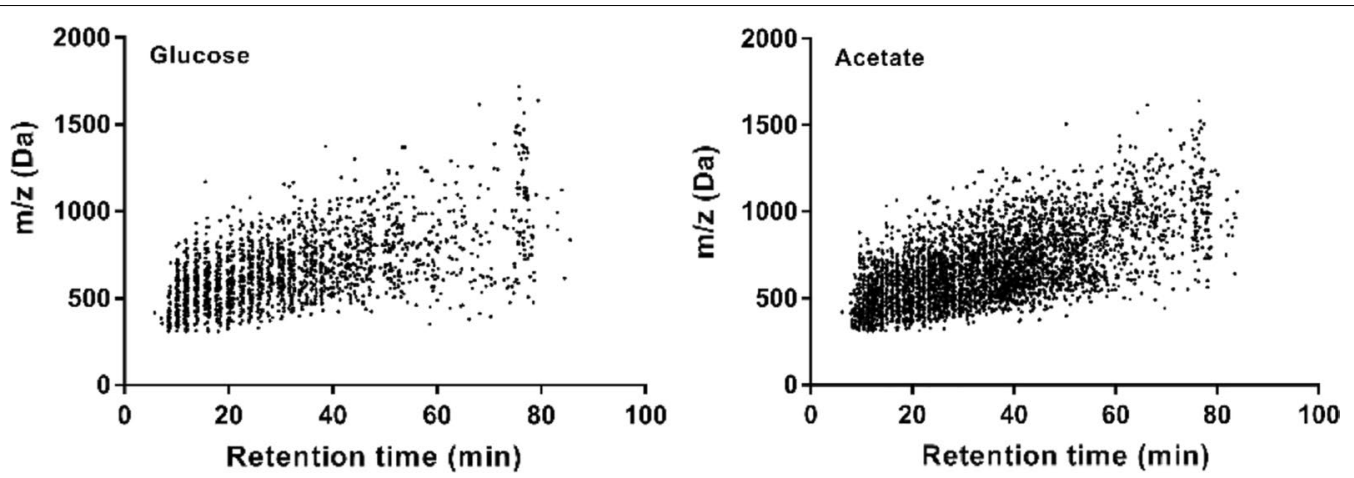

Fig. 2 Scatter plot indicates the mass versus retention time of the peptides identified from glucose-grown and acetate-grown C. glabrata. Most of the separated peptides from these two growth conditions had masses between 300 and $1500 \mathrm{Da}$, and most of the separated peptides eluting from 10 to $80 \mathrm{~min}$

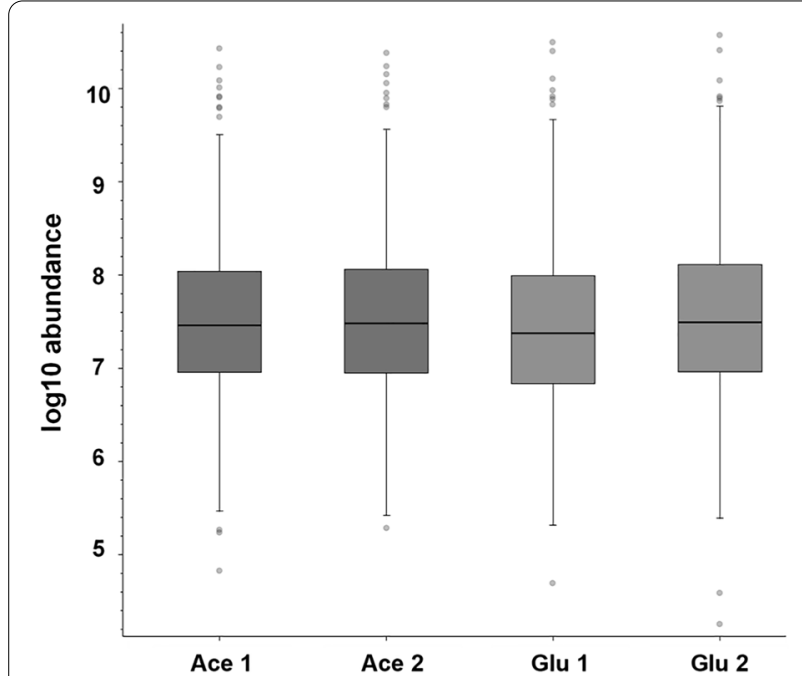

Fig. 3 Abundance and detection range of the proteins identified from glucose-grown and acetate-grown C. glabrata. Highly abundance proteins identified were up to 5 -log higher abundance in comparison to the lowest abundance proteins identified

all up-regulated. Similarly, the gluconeogenic enzymes fructose 1,6-bisphosphatase and phosphoenolpyruvate carboxykinase were up-regulated 3.20-fold and 4.20-fold, respectively. This increase in gluconeogenesis and the glyoxylate cycle reflects the involvement of these pathways in the production of hexoses and pentoses from acetate (e.g. for cell wall biosynthesis and nucleotide biosynthesis, respectively). Also, acetyl-CoA synthetase, an important enzyme involved in acetate metabolism was also up-regulated (1.54-fold) in response to acetate. In addition, proteins potentially associated with the production of anaplerotic precursors for glucose, such as the pyruvate dehydrogenase complex, alcohol dehydrogenase and alanine-glyoxylate transaminase, were also induced.

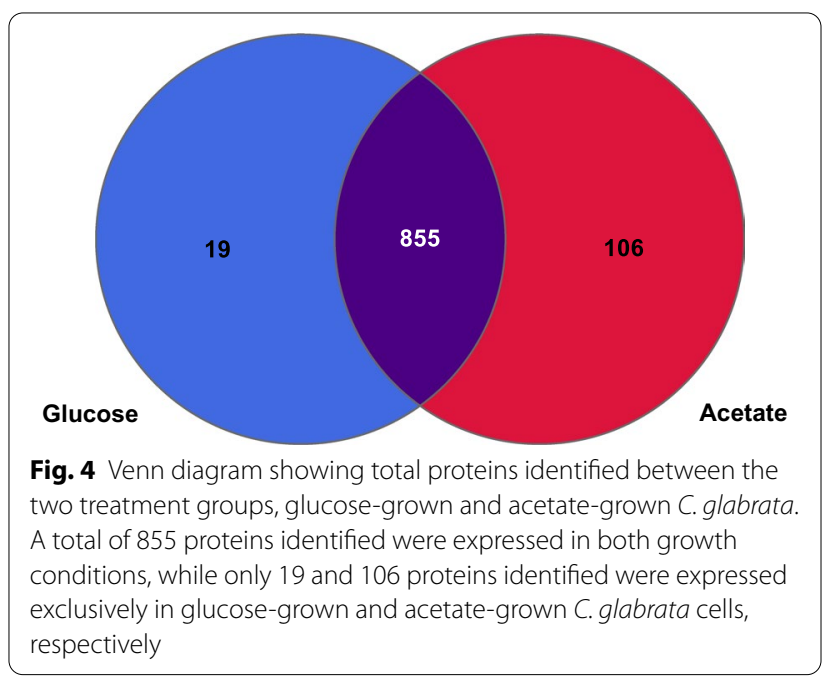

\section{Discussion}

The main goal of this study is to decipher a global view of the metabolic response of the major fungal pathogen C. glabrata, in the presence of acetate as the sole carbon source, through a combination of high-throughput transcriptomic and proteomic profiling. Historically, C. glabrata belongs to the Saccharomyces clade and is phylogenetically more closely related to $S$. cerevisiae than other Candida species [35]. However, further examination reveals that C. glabrata does not act exactly like the baker's yeast. For instance, the capability of carbon assimilation has been reduced significantly in comparison to S. cerevisiae. In fact, C. glabrata is unable to utilize many carbon sources such as galactose, maltose, lactose, sucrose and raffinose and mainly rely on glucose and trehalose [36], it has been shown to be able to utilize certain alternative carbon sources such as carboxylic acids, fatty acids and alcohols [13]. Furthermore, adaptation to these alternative carbon 


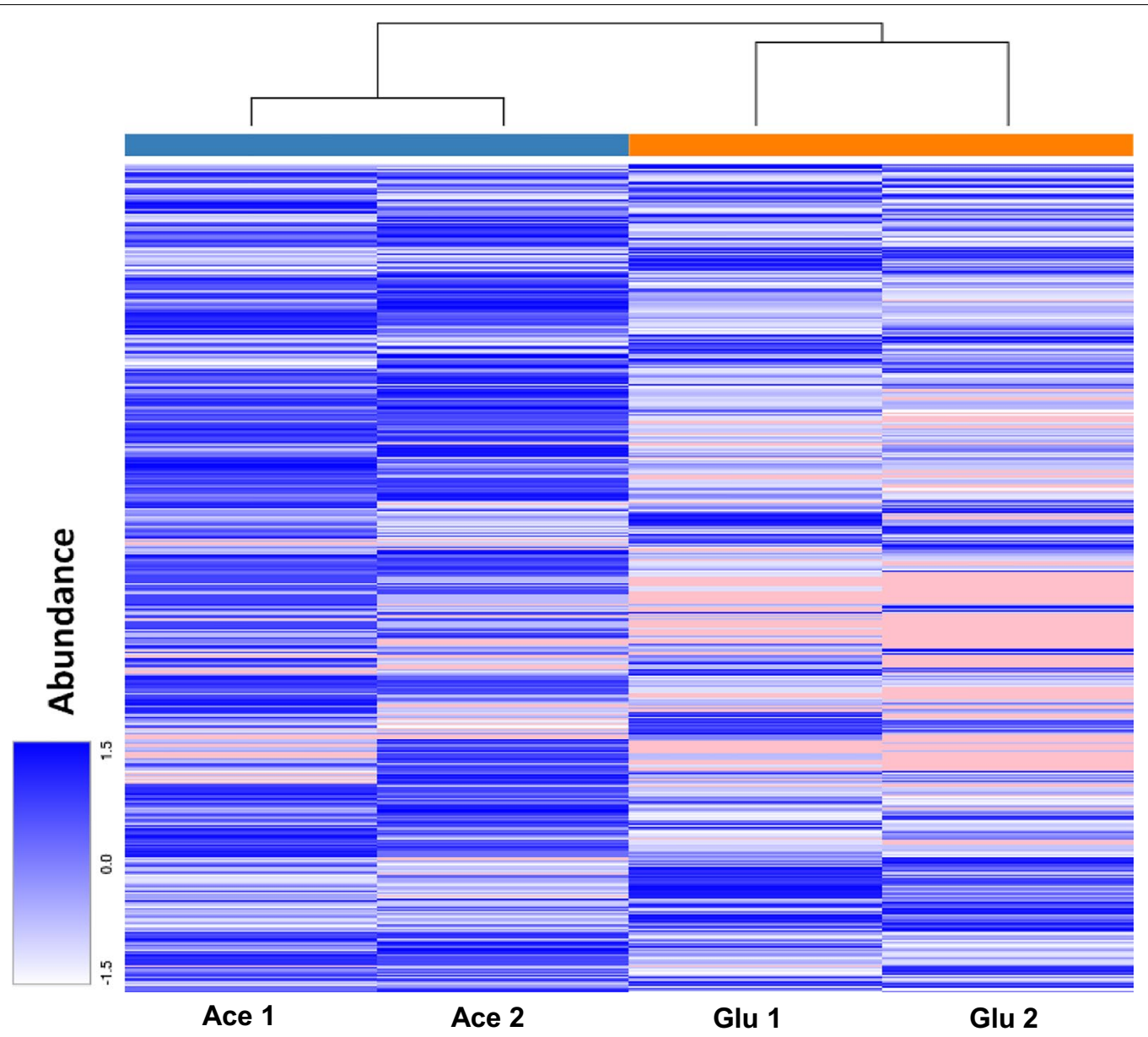

Fig. 5 Hierarchical clustering analysis of the proteins identified from glucose-grown and acetate-grown C. glabrata cells. Hierarchical clustering analysis demonstrated a clear divergent in the proteomes of the glucose-grown and acetate-grown C. glabrata cells, with only minimal differences between the biological replicates

sources also induced physiological changes associated with pathogenicity of C. glabrata. Although C. glabrata is not commonly used in biotechnology field, it is an important microorganism used for cost-effective industrial production of pyruvate, from carbon sources like glucose, acetate and oxaloacetate [37-39].

In human, acetate is present in the blood at the range of 0.1-0.5 mM [40] and has been shown to be relevant within macrophages and neutrophils [5, 15]. Previously, it has been shown that C. glabrata is able to utilize acetate $[9,12,41]$. In addition, putative acetate permease gene $A D Y 2$ was highly induced in macrophagesengulfed $C$. glabrata, signifying the importance of acetate in the survival of engulfed-C. glabrata. Here, it was demonstrated that C. glabrata attunes the alternative carbon metabolism in response to acetate through upregulation and downregulation of key metabolic pathways at both the protein and transcript levels (Fig. 6).
The data suggest that $C$. glabrata regulates its carbon metabolism in favor of production of hexoses during glucose starvation. The majority of the induced pathways in acetate-grown C. glabrata were associated with alternative carbon metabolism, namely gluconeogenesis and the glyoxylate cycle. In fact, C. glabrata appears to employ a pattern of carbon adaptation that resembles other fungal pathogens such as C. albicans, Candida orthopsilosis (a subspecies of C. parapsilosis complex), C. neoformans and Paracoccidioides brasiliensis during glucose starvation $[4,19,42]$. Phagocytosed C. albicans and $C$. neoformans cells appear to encounter glucose deprivation following phagocytosis by the immune cells, and they counteract this stress by replenishing the glucose intermediates from alternative carbon sources, probably through upregulation of genes from the three interconnected pathways: $\beta$-oxidation of fatty acids, the glyoxylate cycle and gluconeogenesis [442] Also, carbon-starved P. brasiliensis induces a metabolic reprogramming that 
Table 4 List of enriched Kyoto encyclopedia of genes and genomes (KEGG) pathways in the differentially expressed proteins (DEPs) of acetate-grown C. glabrata

\begin{tabular}{|c|c|c|c|}
\hline Pathway & Count & Frequency (\%) & EASE score \\
\hline \multicolumn{4}{|l|}{ Up-regulated } \\
\hline Metabolic pathways & 60 & 35.7 & $1.50 \mathrm{E}-12$ \\
\hline Biosynthesis of secondary metabolites & 34 & 20.2 & $1.00 \mathrm{E}-08$ \\
\hline Carbon metabolism & 19 & 11.3 & $1.50 \mathrm{E}-07$ \\
\hline Citrate cycle (TCA cycle) & 11 & 6.5 & $2.40 \mathrm{E}-07$ \\
\hline Biosynthesis of antibiotics & 26 & 15.5 & $6.50 \mathrm{E}-07$ \\
\hline Oxidative phosphorylation & 14 & 8.3 & $5.30 \mathrm{E}-06$ \\
\hline Pyruvate metabolism & 9 & 5.4 & $9.60 \mathrm{E}-05$ \\
\hline Biosynthesis of amino acids & 16 & 9.5 & $1.30 \mathrm{E}-04$ \\
\hline 2-Oxocarboxylic acid metabolism & 8 & 4.8 & $7.80 \mathrm{E}-04$ \\
\hline Glyoxylate and dicarboxylate metabolism & 6 & 3.6 & $2.00 \mathrm{E}-03$ \\
\hline Glycolysis/gluconeogenesis & 7 & 4.2 & $1.80 \mathrm{E}-02$ \\
\hline Pentose and glucuronate interconversions & 3 & 1.8 & $2.80 \mathrm{E}-02$ \\
\hline Arginine biosynthesis & 4 & 2.4 & $3.40 \mathrm{E}-02$ \\
\hline Alanine, aspartate and glutamate metabolism & 4 & 2.4 & $9.40 \mathrm{E}-02$ \\
\hline \multicolumn{4}{|l|}{ Down-regulated } \\
\hline Metabolic pathways & 14 & 29.8 & $6.00 \mathrm{E}-03$ \\
\hline Biosynthesis of antibiotics & 7 & 14.9 & $1.90 \mathrm{E}-02$ \\
\hline Cysteine and methionine metabolism & 3 & 6.4 & $5.30 \mathrm{E}-02$ \\
\hline Biosynthesis of secondary metabolites & 7 & 14.9 & $7.20 \mathrm{E}-02$ \\
\hline
\end{tabular}

mirrors C. albicans and C. neoformans, resulted in drastic changes in the alternative carbon metabolism pathways [19].

The glyoxylate cycle is basically a shunt in the TCA cycle, with which it shares many of its metabolic enzymes [45]. The glyoxylate cycle represented one of the most upregulated pathways in C. glabrata during the change of carbon source from glucose to acetate. In general, all transcripts and proteins from the glyoxylate cycle like isocitrate lyase (ICL1), malate synthase (MLS1), aconitase (ACO1) and citrate synthase (CIT1) were highly induced at the transcript level (1.63- to 4.58-fold) and protein level (2.69- to 6.64-fold), respectively. In addition, fumarate hydratase was also induced in acetate-grown $C$. glabrata. Glyoxylate cycle bypasses the two decarboxylation steps in TCA cycle and allows utilization of twocarbon compounds acetyl-CoA, which is converted from the assimilated acetate by acetyl-CoA synthetase [46]. In this way, flux through the glyoxylate cycle promotes carbon assimilation during the utilization of alternative carbon sources, whilst flux through the TCA cycle maximizes energy generation. It is worth noting that acetylCoA synthetase was also up-regulated in acetate-grown C. glabrata cells. Ultimately, the net oxaloacetate produced from the two-carbon compounds (e.g., acetate) can serve as an intermediate to replenish the TCA cycle in the absence of glucose, or fuel gluconeogenesis for production of glucose intermediate and cellular building blocks [7, 47]. Increased expression of the glyoxylate cycle has been reported in macrophage-engulfed $C$. glabrata [5]. However, this observation could be merely attributed by the general stresses imposed on the fungal cells by the phagocytes. Results obtained strongly suggested that $C$. glabrata could potentially utilize acetate within macrophages, and the presence of acetate clearly induced the glyoxylate cycle and gluconeogenesis.

Generally, C. glabrata employs a carbon flow via classical metabolic pathways such as gluconeogenesis, the glyoxylate cycle and TCA cycle following acetate assimilation. Moreover, suppression of glycolytic growth is a hallmark sign of glucose deprivation in C. glabrata [5]. Other metabolic pathways that were most affected also include nucleotide and amino acid metabolism. In this study, biosynthesis of amino acids including serine (SER2), glycine (GCV1), methionine (MET12, MET15) and isoleucine (ILV1) were all down-regulated, potentially to conserve energy amidst glucose starvation. In addition, L-alanine:2-oxoglutarate aminotransferase was induced in acetate-grown C. glabrata, thus facilitates the conversion of alanine to pyruvate, an intermediate for the glyoxylate shunt. Breakdown of these glucogenic amino acids are likely are being used to produce precursors needed for alternative carbon metabolism in many fungal pathogens [48]. Genes associated with pentose phosphate 
Table 5 Statistically significant differential protein expressions associated with carbon metabolism in acetate-grown $C$. glabrata (q value $<0.05$ )

\begin{tabular}{|c|c|c|c|}
\hline Systematic name & Proteins & Description & $\begin{array}{l}\text { Fold } \\
\text { change } \\
(\log 2)\end{array}$ \\
\hline CAGLOJ03058g & $|c| 1$ & Isocitrate lyase & 6.64 \\
\hline CAGL0D06424g & Acol 1 & Aconitate hydratase & 6.64 \\
\hline CAGLOK12518g & Agx1 & Alanine-glyoxylate transaminase & 6.64 \\
\hline CAGLOL05478g & Rpe1 & Ribulose-phosphate 3-epimerase Transaldolase & 6.64 \\
\hline CAGLOL12254g & Alt1 & L-Alanine:2-oxoglutarate aminotransferase & 6.64 \\
\hline CAGLOH06853g & Adh6 & NADP-dependent alcohol dehydrogenase VI & 6.64 \\
\hline CAGLOH06633g & Pck1 & Phosphoenolpyruvate carboxykinase & 4.20 \\
\hline CAGLOB03663g & Cit1 & Citrate synthase & 3.30 \\
\hline CAGLOH04939g & Fbp1 & Fructose 1,6-bisphosphatase & 3.20 \\
\hline CAGLOC03223g & Sdh2 & Succinate dehydrogenase & 2.62 \\
\hline CAGLOL03982g & Mls1 & Malate synthase & 2.59 \\
\hline CAGLOJ10186g & Lat1 & Pyruvate dehydrogenase complex & 2.33 \\
\hline CAGLOB04917g & ldp2 & Isocitrate dehydrogenase & 2.30 \\
\hline CAGLOL06842g & Thi3 & RNA polymerase II activating transcription factor & 1.91 \\
\hline CAGLOK04235g & Tal1 & Transaldolase & 1.87 \\
\hline CAGLOL12078g & Pda1 & Pyruvate dehydrogenase (acetyl-transferring) & 1.73 \\
\hline CAGLOI07139g & Lsc1 & Succinate-CoA ligase (ADP-forming) & 1.65 \\
\hline CAGLOA01045g & Fum1 & Fumarate hydratase & 1.60 \\
\hline CAGLOL00649g & Acs1 & Acetyl-coenzyme A synthetase & 1.54 \\
\hline CAGLOL05236g & Mdh1 & Malate dehydrogenase & 1.53 \\
\hline CAGL0G02673g & ldh1 & Isocitrate dehydrogenase & 1.50 \\
\hline
\end{tabular}

Data of differential protein expressions ( $q$ value $<0.05$ ) was obtained from proteomic analysis. Fold change (log2) was calculated based on the protein expression of acetate-grown C. glabrata to glucose-grown C. glabrata (control)

pathway such as PRS2, PRS5 and SHB17 and proteins such as ribulose-phosphate 3-epimerase and transaldolase were also down-regulated in response to acetate utilization, thus limiting the nucleotide biosynthetic process in C. glabrata.

Oxidative phosphorylation was also induced in acetategrown C. glabrata at protein and transcripts levels. The process of oxidative phosphorylation takes place in the mitochondria and is responsible for generation of the primary energy source, adenosine triphosphate (ATP) [49]. In this study, acetate utilization via the glyoxylate cycle possibly replenishes the TCA cycle intermediate (i.e., oxaloacetate) and also produces cofactors such as nicotinamide adenine dinucleotide (NADH) and flavin adenine dinucleotide $\left(\mathrm{FADH}_{2}\right)$. Up-regulation of oxidative phosphorylation in acetate-grown C. glabrata suggested that this fungal pathogen could generate ATP required for its survival and the anabolic metabolism from NADH and $\mathrm{FADH}_{2}$ via oxidative phosphorylation in the electron transport chain system.

Production of glucose from glucose intermediates also requires a functional gluconeogenesis. In this study, two metabolic enzymes exclusive to gluconeogenesis, fructose 1,6-biphosphatase (FBP1) and phosphoenolpyruvate carboxykinase ( $P C K 1)$ were induced 7.35- and 5.64-fold at the transcript level, as well as 3.20- and 4.20-fold at the protein level, respectively (Tables 3,5). Both of these enzymes contribute to the conversion of oxaloacetate from the glyoxylate cycle into hexose phosphates via gluconeogenesis. Catabolism and anabolism of glucose in fungi are controlled by glycolysis and gluconeogenesis, respectively [50]. In the absence of glucose, catabolism of glucose via glycolysis appears to be repressed, as demonstrated by the down-regulation of transcripts of many glycolytic enzymes such as phosphofructokinase (PFK1 and $P F K 2$ ), hexokinase (HXK2), and pyruvate kinase (CDC19). However, these glycolytic enzymes were not detected at the protein level in this study.

Other than the glyoxylate cycle and gluconeogenesis enzymes, the transcript of the TCA cycle enzymes such as isocitrate dehydrogenase (IDP2), succinate dehydrogenase $(S D H 2)$ and $\alpha$-ketoglutarate dehydrogenase complex (KGD1, KGD2) were up-regulated in the absence of glucose. It has been reported that a subset of $C$. albicans within the cell populations activated the glyoxylate cycle and gluconeogenic growth during early phagocytosis by 


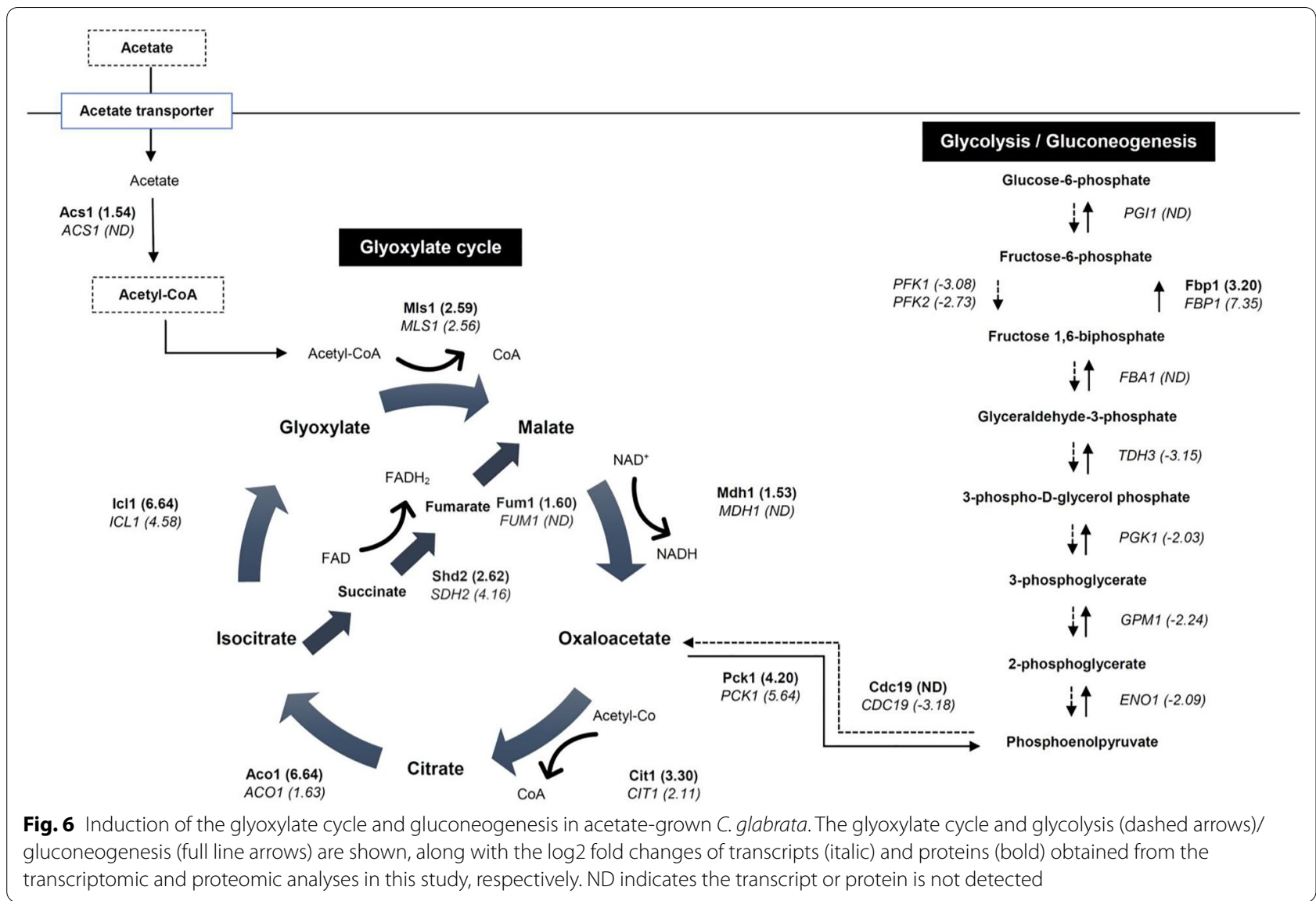

the immune cells. However, they also expressed glycolytic mechanisms that are required for the systemic disease progression [43]. The same reasoning might also be applicable to acetate-grown C. glabrata. The fungal pathogen could potentially express TCA cycle enzymes concurrently with gluconeogenic enzymes in preparation for the colonization of the host, once glucose availability is no longer a limiting factor.

Mitogen-activated protein kinase pathways are conserved signalling cascades in eukaryotes that are involved in the transduction of extracellular or environmental stimuli for transcriptional regulation [51, 52]. In fungi, MAPK signalling pathways have been implicated to be involved in a wide range of processes such as fungal pathogenicity, mating, morphogenesis, stress resistance and cell wall integrity [53-55]. In this study, MAPK signalling components were also up-regulated in acetategrown C. glabrata (STE11, STE12, STE20, SLN1, YPD1, SSK1, SLT2, SWI4, MID2, MSN2, MSG5, BEM1 and MCM1) compared to glucose-grown cells (Table 2). The STE vegetative pathway has been reported to play an important role in the maintenance of cell wall integrity of S. cerevisiae via Ste11, Ste12 (with the DNA binding protein Mcm1) and Ste20, and these transcription factors are responsible for the transcription of genes related to the conservation of cell wall integrity [56-58]. In C. glabrata, although both Ste11 and Ste12 are largely expendable for the cell wall integrity (unlike in S. cerevisiae), Ste20 is crucial in maintaining the cell wall integrity of C. glabrata [59-61]. Similarly, increased C. glabrata SLT2 expression has been linked to the elevated chitin level and echinocandin tolerance in C. glabrata [62], signifying the importance of SLT2 and its downstream transcriptional regulator (e.g. Swi4) in the rescue of cell wall integrity and tolerance to cell-damaging stresses such as Congo red, Calcofluor white, caspofungin and micafungin [62-64].

The orthologous gene MID2 was also induced in acetate-grown cells. This implies that $C$. glabrata could activate $P K C 1-M P K 1$ signal transduction pathway via MID2 similar to $S$. cerevisiae for maintenance of cell wall integrity [65]. The high-osmolarity glycerol (HOG) pathway, which is normally inactivated by three phospho-relay components Sln-Ypd1-Ssk1 under normal 
condition mediates cellular response to osmotic shocks [66]. It is worth mentioning that SLN1, YPD1 and SSK1 were induced in acetate-grown $C$. glabrata, suggesting that HOG pathway is likely inactivated in this condition. Taken together, results obtained highly suggest that the fungal cells may employ MAPK signalling to control its transcriptional regulation in order to modulate fitness attributes of C. glabrata in response to acetate, as demonstrated in our study [13], particularly on cell wall structure, stress and antifungal resistance. Regulation of fitness and pathogenic attributes via MAPK pathways could potentially assist in C. glabrata ability to resist phagocytic killing [67-69].

In addition, ethanol catabolism $(A D H 2, A L D 5)$ were also inhibited in the presence of acetate as the sole carbon source. Alcohol dehydrogenase $(A D H 2)$ catalyses the breakdown of ethanol into acetaldehyde, which could be converted to acetate by aldehyde dehydrogenase (ALD5) $[46,70]$. It is worth to highlight that minor discrepancies between transcript and protein abundances were observed in pathways associated with biosynthesis of secondary metabolites and amino acids. Biosynthesis of amino acids such as valine, leucine, isoleucine, phenylalanine, tyrosine, tryptophan, cysteine, methionine, histidine and arginine were all repressed at the transcript levels, whilst protein abundance levels were increased. Differences between transcriptome and proteome data could be attributed, at least in part to the translational regulation. In fact, there were precedents for mRNAs associated with amino acid biosynthesis such as CPA1 and GCN4 to be translationally regulated in yeast cells $[71,72]$.

\section{Conclusion}

Overall, RNA-sequencing and label-free quantitative proteomic analyses had successfully revealed the first transcriptomic and proteomic responses of C. glabrata in the presence of acetate as the sole carbon source. Here, we showed that C. glabrata mainly depended on the glyoxylate cycle and gluconeogenic growth to replenish glucose intermediates and generate energy from acetate. This results also strengthen the idea that $C$. glabrata could potentially survive within macrophages, owing to its ability to switch into alternative carbon metabolism. In addition, investigation of the reprogramming of carbon metabolism in C. glabrata is imperative to understand the metabolic regulation and pathogenesis of this fungal pathogen in glucose deprivation condition. It is worth mentioning that only a single strain of C. glabrata and carbon source were analyzed and this is a limitation of the study. Therefore, potential future study focuses on clinical strains of C. glabrata and other physiologically relevant carbon sources are certainly warranted.

\section{Supplementary Information}

The online version contains supplementary material available at https://doi. org/10.1186/s12929-020-00700-8.

Additional file 1: Table S1. List of primers used in qPCR experiments. Table S2. Coefficient of determination $\left(r^{2}\right)$ between biological replicates for glucose- and acetate-grown C. glabrata. Table S3. List of enriched GO terms associated with up-regulated DEGs of acetate-grown C. glabrata cells. Table S4. List of enriched GO terms associated with down-regulated DEGs of acetate-grown C. glabrata cells. Table S5. List of enriched GO terms associated with DEPs of acetate-grown C. glabrata.

\section{Abbreviations}

AGC: Automatic gain control; ATCC: American Type Culture Collection; ATP: Adenosine triphosphate; CHAPS: 3-[(3-Cholamidopropyl)dimethylammonio]1-propanesulfonate; CT: Cycle threshold; DAVID: Database for Annotation, Visualization and Integrated Discovery; DEGs: Differentially expressed genes; DEPs: Differentially expressed proteins; DTT: Dithiothreitol, ENA: European Nucleotide Achieve; $\mathrm{FADH}_{2}$ : Flavin adenine dinucleotide (hydroquinone form); FDR: False discovery rate; FPKM: Fragments per kilobase of transcript per million mapped fragments; GO: Gene ontology; HCD: High-energy collisional dissociation; HOG: High-osmolarity glycerol; iTRAQ: Isobaric tagging for relative and absolute quantification; KEGG: Kyoto Encyclopedia of Genes and Genomes; MAPK: Mitogen-activated protein kinase; NADH: Nicotinamide adenine dinucleotide (reduced form); NCAC: Non-Candida albicans Candida; NCBI: National Center for Biotechnology Information; NGS: Next generation sequencing; PBS: Phosphate-buffered saline; SC: Synthetic complete; SPE: Solid phase extraction; TCA: Tricarboxylic acid; TCEP: Tris(2-carboxyethyl) phosphine; UHPLC: Ultra-high-performance liquid chromatography; YNB: Yeast nitrogen base; YPD: Yeast extract peptone dextrose.

\section{Acknowledgements}

Not applicable.

\section{Authors' contributions}

Conceptualization, SY, AB, DS and LT; Methodology, SY, AB, BL, YK, KL, DS and $\mathrm{LT}$; Investigation, SY, BL and HY; Resources, KL, YK and LT; Formal analysis, SY, BL and $L T$; Funding acquisition, $L T$; Writing —original draft preparation, SY; Writing-review and editing, $A B, B L, Y K, K L$ and $L T$. All authors read and approved the final manuscript.

\section{Funding}

This work was supported by Putra Grant_-Putra Graduate Initiative (IPS) from Universiti Putra Malaysia (Grant Number: GPS-IPS/2018/9612400). SC is a recipient of the MyBrain 15 Scholarship from Ministry of Education, Malaysia. AB was supported by the UK Medical Research Council (www.mrc.ac.uk: MR/ M026663/1), the Medical Research Council Centre for Medical Mycology (MR/ N006364/1), the Wellcome Trust (www.wellcome.ac.uk: 097377), and the European Commission (FunHoMic: H2020-MSCA-ITN-2018-812969).

\section{Availability of data and materials}

The datasets supporting the conclusions of this article are available in the (1) European Nucleotide Archive (RNA-sequencing data, study accession number: PRJEB33880) and (2) ProteomeXchange Consortium via the PRIDE partner repository (proteomics data, dataset identifier: PXD014916 and 10.6019/ PXD014916).

Ethics approval and consent to participate Not applicable.

Consent for publication

Not applicable. 


\section{Competing interests}

The authors declare that they have no competing interests.

\section{Author details}

${ }^{1}$ Department of Medical Microbiology, Faculty of Medicine and Health Sciences, Universiti Putra Malaysia, 43400 Serdang, Selangor, Malaysia. ${ }^{2}$ MRC Centre for Medical Mycology, University of Exeter, Geoffrey Pope Building, Stocker Road, Exeter EX4 4QD, UK. ${ }^{3}$ Proteomics and Metabolomics (PROMET) Group, Malaysian Palm Oil Board, Bandar Baru Bangi, 43000 Kajang, Selangor, Malaysia. ${ }^{4}$ Department of Biomedical Sciences, Faculty of Medicine and Health Sciences, Universiti Putra Malaysia, 43400 Serdang, Selangor, Malaysia. ${ }^{5}$ Department of Pathology, Faculty of Medicine and Health Sciences, Universiti Putra Malaysia, 43400 Serdang, Selangor, Malaysia. ${ }^{6}$ Infectomics Cluster, Advanced Medical and Dental Institute, Universiti Sains Malaysia, 13200 Kepala Batas, Pulau Pinang, Malaysia. ${ }^{7}$ Department of Medical Laboratory Science, Faculty of Allied Health Sciences, Bayero University, Kano, Nigeria.

Received: 28 June 2020 Accepted: 21 December 2020 Published online: 02 January 2021

\section{References}

1. Pfaller MA, Andes DR, Diekema DJ, Horn DL, Reboli AC, Rotstein C, et al. Epidemiology and outcomes of invasive candidiasis due to non-albicans species of Candida in 2496 patients: data from the Prospective Antifungal Therapy (PATH) registry 2004-2008. PLoS ONE. 2014;9:e101510.

2. Kullberg BJ, Arendrup M. Invasive candidiasis. N Engl J Med. 2015;373:1445-56.

3. Sobel JD. The emergence of non-albicans Candida species as cause of invasive candidiasis and candidemia. Curr Infect Dis Rep. 2016:8:427-33.

4. Lorenz MC, Bender JA, Fink GR. Transcriptional response of Candida albicans upon internalization by macrophages. Eukaryot Cell. 2004;3:1076-87

5. Kaur R, Ma B, Cormack BP. A family of glycosylphosphatidylinositol-linked aspartyl proteases is required for virulence of Candida glabrata. Proc Natl Acad Sci USA. 2007;104:7628-33.

6. Ramírez MA, Lorenz MC. Mutations in alternative carbon utilization pathways in Candida albicans attenuate virulence and confer pleiotropic phenotypes. Eukaryot Cell. 2007;6:280-90.

7. Rai MN, Balusu S, Gorityala N, Dandu L, Kaur R. Functional genomic analysis of Candida glabrata-macrophage interaction: role of chromatin remodelling in virulence. PLoS Pathog. 2012;8:e1002863.

8. Ueno K, Matsumoto Y, Uno J, Sasamoto K, Sekimizu K, Kinjo Y, et al. Intestinal resident yeast Candida glabrata requires Cyb2p-mediated lactate assimilation to adapt in mouse intestine. PLOS ONE. 2011;6:e24759.

9. Cunha DV, Salazar SB, Lopes MM, Mira NP. Mechanistic insights underlying tolerance to acetic acid stress in vaginal Candida glabrata clinical isolates. Front Microbiol. 2017;8:259.

10. Ene IV, Adya AK, Wehmeier S, Brand AC, MacCallum DM, Gow NA, et al. Host carbon sources modulate cell wall architecture, drug resistance and virulence in a fungal pathogen. Cell Microbiol. 2012;14:1319-35.

11. Brown AJ, Brown GD, Netea MG, Gow NA. Metabolism impacts upon Candida immunogenicity and pathogenicity at multiple levels. Trends Microbiol. 2014:22:614-22.

12. Mota S, Alves R, Carneiro C, Silva S, Brown AJ, Istel F, et al. Candida glabrata susceptibility to antifungals and phagocytosis is modulated by acetate. Front Microbiol. 2015;6:919.

13. Chew SY, Ho KL, Cheah YK, Sandai D, Brown A, Than LTL. Physiologically relevant alternative carbon sources modulate biofilm formation, cell wall architecture, and the stress and antifungal resistance of Candida glabrata. Int J Mol Sci. 2019;20:E3172

14. Dujon B, Sherman D, Fischer G, Durrens P, Casaregola S, Lafontaine I, et al. Genome evolution in yeasts. Nature. 2014;430:35-44.

15. Fukuda Y, Tsai HF, Myers TG, Bennett JE. Transcriptional profiling of Candida glabrata during phagocytosis by neutrophils and in the infected mouse spleen. Infect Immun. 2013;81:1325-33.

16. Goodwin S, McPherson JD, McCombie WR. Coming of age: ten years of next-generation sequencing technologies. Nat Rev Genet. 2016;17:333-51.
17. Nagalakshmi U, Wang Z, Waern K, Shou C, Raha D, Gerstein M, et al. The transcriptional landscape of the yeast genome defined by RNA sequencing. Science. 2008;320:1344-9.

18. Chen Y, Toffaletti DL, Tenor JL, Litvintseva AP, Fang C, Mitchell TG, et al. The Cryptococcus neoformans transcriptome at the site of human meningitis. mBio. 2014;5:e01087-e1113

19. Lima P, Casaletti L, Bailão AM, de Vasconcelos AT, Fernandes G, Soares CM. Transcriptional and proteomic responses to carbon starvation in Paracoccidioides. PLoS Negl Trop Dis. 2014;8:e2855.

20. Wang T, Xiu J, Zhang Y, Wu J, Ma X, Wang Y, et al. Transcriptional responses of Candida albicans to antimicrobial peptide MAF-1A. Front Microbiol. 2017;8:894.

21. Wang Z, Gerstein M, Snyder M. RNA-Seq: a revolutionary tool for transcriptomics. Nat Rev Genet. 2009;10:57-63.

22. Dai W, Chen Q, Wang Q, White RR, Liu J, Liu H. Complementary transcriptomic and proteomic analyses reveal regulatory mechanisms of milk protein production in dairy cows consuming different forages. Sci Rep. 2017;7:44234.

23. Neilson KA, Ali NA, Muralidharan S, Mirzaei M, Mariani M, Assadourian $G$, et al. Less label, more free: approaches in label-free quantitative mass spectrometry. Proteomics. 2011;11:535-53.

24. Trinh HV, Grossmann J, Gehrig P, Roschitzki B, Schlapbach R, Greber UF, et al. iTRAQ-based and label-free proteomics approaches for studies of human adenovirus Infections. Int J Proteomics. 2013;2013:581862.

25. Latosinska A, Vougas K, Makridakis M, Klein J, Mullen W, Abbas M, et al. Comparative analysis of label-free and 8-Plex iTRAQ approach for quantitative tissue proteomic analysis. PLoS ONE. 2015;10:e0137048.

26. Wang X, Liu Q, Zhang B. Leveraging the complementary nature of RNASeq and shotgun proteomics data. Proteomics. 2014;14:2676-87.

27. Collart MA, Oliviero S. Preparation of yeast RNA. Curr Protoc Mol Biol. 2001:23:13121-5.

28. Trapnell C, Roberts A, Goff L, Pertea G, Kim D, Kelly DR, et al. Differential gene and transcript expression analysis of RNA-Seq experiments with TopHat and Cufflinks. Nat Protoc. 2012;7:562-78.

29. Trapnell C, Williams BA, Pertea G, Mortazavi A, Kwan G, van Baren MJ, et al. Transcript assembly and quantification by RNA-Seq reveals unannotated transcripts and isoform switching during cell differentiation. Nat Biotechnol. 2010:28:511-5.

30. Harrison PW, Alako B, Amid C, Cerdeño-Tárraga A, Cleland I, Holt S, et al. The European nucleotide archive in 2018. Nucleic Acids Res. 2018;47:D84-8

31. Huang DW, Sherman BT, Lempicki RA. Systematic and integrative analysis of large gene lists using DAVID bioinformatics resources. Nat Protoc. 2008:4:44-57.

32. Aoki W, Ueda T, Tatsukami Y, Kitahara N, Morisaka H, Kuroda K, et al. Timecourse proteomic profile of Candida albicans during adaptation to a fetal serum. Pathog Dis. 2013:67:67-75.

33. Lau BY, Clerens S, Morton JD, Dyer JM, Deb-Choudhury S, Ramli U. Application of a mass spectrometric approach to detect the presence of fatty acid biosynthetic phosphopeptides. Protein J. 2016;35:163-70.

34. Perez-Riverol Y, Csordas A, Bai J, Bernal-Llinares M, Hewapathirana S, Kundu DJ, et al. The PRIDE database and related tools and resources in 2019: improving support for quantification data. Nucleic Acids Res. 2019;47:D442-5.

35. Papon N, Courdavault V, Clastre M, Bennett RJ. Emerging and emerged pathogenic Candida species: beyond the Candida albicans paradigm. PLoS Pathog. 2013;9:e1003550.

36. Bolotin-Fukuhara M, Fairhead C. Candida glabrata: a deadly companion? Yeast. 2014;31:279-88.

37. Liu L, Du G, Li Y, Li H, Chen J. Enhancement of pyruvate production by Torulopsis glabrata through supplement of oxaloacetate as carbon source. Biotechnol Bioprocess Eng. 2005;10:136-41.

38. Li S, Chen X, Liu L, Chen J. Pyruvate production in Candida glabrata: manipulation and optimization of physiological function. Crit Rev Biotechnol. 2013;36:1-10.

39. Xu N, Liu L, Zou W, Liu J, Hu Q, Chen J. Reconstruction and analysis of the genome-scale metabolic network of Candida glabrata. Mol BioSyst. 2013;9:205.

40. Knowles SE, Jarrett IG, Filsell OH, Ballard FJ. Production and utilization of acetate in mammals. Biochem J. 1974;142:401-11. 
41. Liu LM, Li Y, Li HZ, Chen J. Manipulating the pyruvate dehydrogenase bypass of a multi-vitamin auxotrophic yeast Torulopsis glabrata enhanced pyruvate production. Lett Appl Microbiol. 2004;39:199-206.

42. Fan W, Kraus PR, Boily MJ, Heitman J. Cryptococcus neoformans gene expression during murine macrophage infection. Eukaryot Cell. 2005;4:1420-33.

43. Barelle CJ, Priest CL, MacCallum DM, Gow NA, Odds FC, Brown AJ. Nichespecific regulation of central metabolic pathways in a fungal pathogen. Cell Microbiol. 2006;8:961-71.

44. Pires RH, Cataldi TR, Franceschini LM, Labate MV, Fusco-Almeida AM, Labate CA, et al. Metabolic profiles of planktonic and biofilm cells of Candida orthopsilosis. Future Microbiol. 2016;11:1299-313.

45. Kunze M, Pracharoenwattana I, Smith SM, Hartig A. A central role for the peroxisomal membrane in glyoxylate cycle function. Biochim Biophys Acta. 2006;1763:1441-52.

46. Turcotte $B$, Liang XB, Robert F, Soontorngun N. Transcriptional regulation of nonfermentable carbon utilization in budding yeast. FEMS Yeast Res. 2010;10:2-13.

47. Lorenz MC, Fink GR. Life and death in a macrophage: role of the glyoxylate cycle in virulence. Eukaryot Cell. 2002;1:657-62.

48. Miramón P, Lorenz MC. A feast for Candida: Metabolic plasticity confers an edge for virulence. PLoS Pathog. 2017;13:e1006144.

49. Saraste M. Oxidative phosphorylation at the fin de siècle. Science. 1999;283:1488-93.

50. Ene IV, Brunke S, Brown AJ, Hube B. Metabolism in fungal pathogenesis. Cold Spring Harb Perspect Med. 2014;4:a019695.

51. Monge RA, Román E, Nombela C, Pla J. The MAP kinase signal transduction network in Candida albicans. Microbiol. 2006;152:905-12.

52. De Paula RG, Antoniêto ACC, Carraro CB, Lopes DCB, Persinoti GF, Peres NTA, et al. The duality of the MAPK signaling pathway in the control of metabolic processes and cellulase production in Trichoderma reesei. Sci Rep. 2018;8:14931.

53. Jain R, Valiante V, Remme N, Docimo T, Heinekamp T, Hertweck C, et al. The MAP kinase MpkA controls cell wall integrity, oxidative stress response, gliotoxin production and iron adaptation in Aspergillus fumigatus. Mol Microbiol. 2011;82:39-53.

54. Wang M, Zhao Q, Yang J, Jiang B, Wang F, Liu K, et al. A mitogen-activated protein kinase Tmk3 participates in high osmolarity resistance, cell wall integrity maintenance and cellulase production regulation in Trichoderma reesei. PLoS ONE. 2013;8:e72189.

55. Brown NA, Dos Reis TF, Goinski AB, Savoldi M, Menino J, Almeida MT, et al. The Aspergillus nidulans signalling mucin MsbA regulates starvation responses, adhesion and affects cellulase secretion in response to environmental cues. Mol Microbiol. 2014;94:1103-20.

56. Kuo MH, Nadeau ET, Grayhack EJ. Multiple phosphorylated forms of the Saccharomyces cerevisiae $\mathrm{Mcm} 1$ protein include an isoform induced in response to high salt concentrations. Mol Cell Biol. 1997;17:819-32.

57. Lee BN, Elion EA. The MAPKKK Ste11 regulates vegetative growth through a kinase cascade of shared signaling components. Proc Natl Acad Sci USA. 1999;96:12679-84.

58. Cullen PJ, Schultz J, Horecka J, Stevenson BJ, Jigami Y, Sprague GF Jr. Defects in protein glycosylation cause SHO1-dependent activation of a STE12 signaling pathway in yeast. Genetics. 2000;155:1005-18.

59. Calcagno AM, Bignell E, Warn P, Jones MD, Denning DW, Mühlschlegel FA, et al. Candida glabrata STE 12 is required for wild-type levels of virulence and nitrogen starvation induced filamentation. Mol Microbiol. 2003;50:1309-18.

60. Calcagno AM, Bignell E, Rogers TR, Canedo M, Mühlschlegel FA, Haynes K. Candida glabrata Ste20 is involved in maintaining cell wall integrity and adaptation to hypertonic stress, and is required for wild-type levels of virulence. Yeast. 2004;21:557-68.

61. Calcagno AM, Bignell E, Rogers TR, Jones MD, Mühlschlegel FA, Haynes K. Candida glabrata Ste11 is involved in adaptation to hypertonic stress, maintenance of wild-type levels of filamentation and plays a role in virulence. Med Mycol. 2005;43:355-64.

62. Cota JM, Grabinski JL, Talbert RL, Burgess DS, Rogers PD, Edlind TD, et al. Increases in SLT2 expression and chitin content are associated with incomplete killing of Candida glabrata by caspofungin. Antimicrob Agents Chemother. 2008;52:1144-6.

63. Miyazaki T, Inamine T, Yamauchi S, Nagayoshi Y, Saijo T, Izumikawa K, et al. Role of the SIt2 mitogen-activated protein kinase pathway in cell wall integrity and virulence in Candida glabrata. FEMS Yeast Res. 2010;10:343-52.

64. Nagayoshi Y, Miyazaki T, Minematsu A, Yamauchi S, Takazono T, Nakamura $\mathrm{S}$, et al. Contribution of the SIt2-regulated transcription factors to echinocandin tolerance in Candida glabrata. FEMS Yeast Res. 2014;14:1128-31.

65. Ketela T, Green R, Bussey H. Saccharomyces cerevisiae mid2p is a potential cell wall stress sensor and upstream activator of the PKC1-MPK1 cell integrity pathway. J Bacteriol. 1999;181:3330-40.

66. Dexter JP, Xu P, Gunawardena J, McClean MN. Robust network structure of the SIn1-Ypd1-Ssk1 three-component phospho-relay prevents unintended activation of the HOG MAPK pathway in Saccharomyces cerevisiae. BMC Syst Biol. 2015;9:17.

67. Seider K, Heyken A, Lüttich A, Miramón P, Hube B. Interaction of pathogenic yeasts with phagocytes: survival, persistence and escape. Curr Opin Microbiol. 2010;13:392-400.

68. Seider K, Brunke S, Schild L, Jablonowski N, Wilson D, Majer O, et al. The facultative intracellular pathogen Candida glabrata subverts macrophage cytokine production and phagolysosome maturation. J Immunol. 2011;187:3072-86.

69. Duggan S, Essig F, Hünniger K, Mokhtari Z, Bauer L, Lehnert T, et al. Neutrophil activation by Candida glabrata but not Candida albicans promotes fungal uptake by monocytes. Cell Microbiol. 2015;17:1259-76.

70. Simpson-Lavy K, Kupiec M. Carbon catabolite repression in yeast is not limited to glucose. Sci Rep. 2019;9:6491.

71. Werner M, Feller A, Messenguy F, Piérard A. The leader peptide of yeast gene CPA1 is essential for the translational repression of its expression. Cell. 1987;49:805-13.

72. Hinnebusch AG. Translational regulation of GCN4 and the general amino acid control of yeast. Annu Rev of Microbiol. 2005;59:407-50.

\section{Publisher's Note}

Springer Nature remains neutral with regard to jurisdictional claims in published maps and institutional affiliations. 